\title{
Analogies are interesting!
}

\author{
K.R.S. Sastry
}

K.R.S. Sastry obtained his Bachelor's and Master's degrees in mathematics from the University of Mysore in India. He taught mathematics, initially in the State of Karnataka in India, later on in Ethiopia. After his retirement in 1994 he returned to the city of Bangalore in India, where he continues to work on mathematical problems.

Let an equilateral triangle $A B C$ be inscribed in a circle. Let $P$ be any point on the minor $\overparen{B C}(\operatorname{arc} B C)$ as in Fig. 1. Then it is well-known that $A P=B P+P C$. (This can be easily proved using Ptolemy's theorem, trigonometry or by other means.) Is not this property pretty? Obviously, we do not expect a non equilateral triangle to exhibit such a property. However, in a given triangle $A B C$ it seems reasonable to expect the existence of at least one point $P$ with the above property. Surprisingly, a non equilateral isosceles triangle does not possess such a point. Our discussion examines the reason and considers interesting special cases.

Next, we look at the right angled triangle $A B C$ in which $\angle B A C=\pi / 2$. If $A D$ is drawn perpendicular to $B C$ (shown in Fig. 2), then it is well-known that $A D^{2}=B D \cdot D C$. In fact, if $D^{\prime}$ is the mid point of $B C$ of the same $\triangle A B C$, then also it trivially follows that $A D^{\prime 2}=B D^{\prime} \cdot D^{\prime} C$ because $D^{\prime}$ is the center of the circle $A B C$. The second part of our discussion focuses on the characterization of $\triangle A B C$ in which at least one point $D$ exists on $B C$ with the property just mentioned. Before we begin the discussion, let us recall the necessary background euclidean geometry results.

Analogien spielen bekanntlich eine wichtige Rolle beim Entdecken neuer Erkenntnisse in der Mathematik. An diese Tatsache knüpft der Autor in der nachfolgenden Arbeit an. Ausgehend von dem bekannten Ergebnis, dass für einen Punkt $P$ auf dem Umkreis eines gleichseitigen Dreiecks $A B C$ zwischen $B$ und $C$ die Beziehung $A P=B P+P C$ besteht, wird in Umkehrung dazu nach notwendigen und hinreichenden Bedingungen an die Seiten eines beliebigen Dreiecks $A B C$ gesucht, so dass ein Punkt $P$ auf dem Umkreis des gegebenen Dreiecks zwischen $B$ und $C$ existiert, der der obigen Gleichung genügt. Ausgangspunkt für das zweite Beispiel ist der Höhensatz im rechtwinkligen Dreieck. Verallgemeinernd dazu wird nach notwendigen und hinreichenden Bedingungen an die Seiten eines beliebigen Dreiecks $A B C$ gefragt, so dass ein Punkt $D$ auf der Seite $B C$ existiert, der die Beziehung $A D^{2}=B D \cdot D C$ erfüllt. 


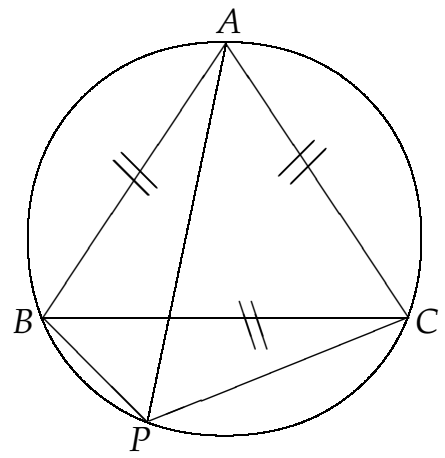

Fig. $1 \quad A P=B P+P C$

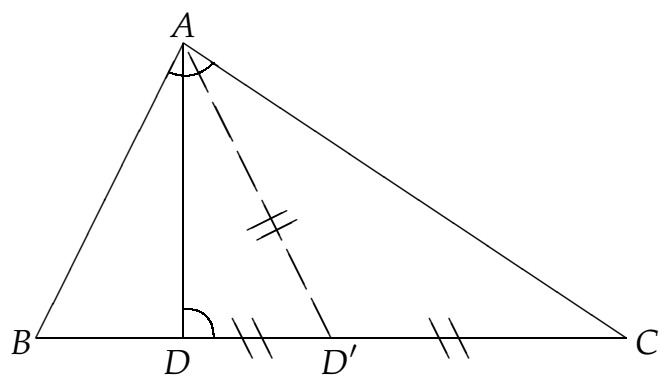

Fig. $2 A D^{2}=B D \cdot D C$ and $A D^{\prime 2}=B D^{\prime} \cdot D^{\prime} C$

\section{Background material}

We use the standard notation throughout our discussion. In $\triangle A B C, a, b, c$ denote either the sides or the lengths $B C, C A, A B$ and $A, B, C$ denote either the vertices or the measures of $\angle B A C, \angle C B A, \angle A C B$, respectively. We assume the reader's familiarity with the angle bisector theorem, properties of similar triangles, intersecting chords of a circle and so on. In addition, we need the result that is sometimes called Stewart's theorem. Therefore, we derive it below as an application of the cosine rule. This result is central to our discussion.

Theorem 0 Let $D$ be a point on the side $B C$ of $\triangle A B C$. If $B D / D C=m / n$, then

$$
A D^{2}=\frac{m b^{2}+n c^{2}}{m+n}-\frac{m n a^{2}}{(m+n)^{2}} .
$$




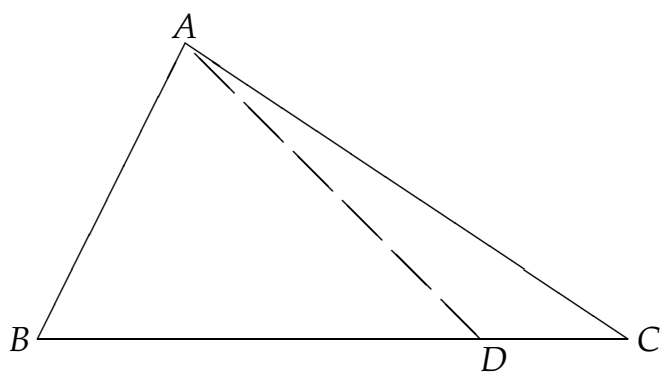

Fig. $3 \frac{B D}{D C}=\frac{m}{n}, A D^{2}=$ ?

Proof. We refer to Fig. 3. If $B D / D C=m / n$, then by the property of equal ratios $B D /(B D+D C)=m /(m+n)$, so $B D=m a /(m+n)$ and $D C=n a /(m+n)$. We now apply the cosine rule for $\triangle A B D$. This gives

$$
\begin{aligned}
A D^{2} & =A B^{2}+B D^{2}-2 \cdot A B \cdot B D \cdot \cos (\angle A B D) \\
& =c^{2}+\frac{m^{2} a^{2}}{(m+n)^{2}}-2 \cdot c \cdot \frac{m a}{m+n} \cdot \frac{c^{2}+a^{2}-b^{2}}{2 c a} .
\end{aligned}
$$

The simplification (left to the reader) of the above expression leads to the result given in $(*)$.

Remark 1 The result $(*)$ is true for any position of the point $D$ on $B C$ that is within $B C$ as in Fig. 3, on $B C$ extended or $C B$ extended. In such an extended case we should observe the usual sign convention, i.e., $B D>0, D C>0$ if $D$ is as in Fig. $3, B D>0$, $D C<0$ if $D$ is to the right of $B C$ and $B D<0, D C>0$ if $D$ is to the left of $B C$.

\section{The analogies}

We first discuss the equilateral triangle analogy mentioned in the introduction. Theorem 1 shows that the sides of a $\triangle A B C$ must be expressible in a certain form for the existence of a point $P$ on the minor $\overparen{B C}$ with the property $A P=B P+P C$. We will then deduce a number of corollaries. Except for Corollary 6, Theorem 1 and the Corollaries 1 to 5 are true for positive real numbers $a, b, c, m$ and $n$.

Theorem 1 Let $\triangle A B C$ be inscribed in a circle. Then a point $P$ exists on the minor $\overparen{B C}$ such that $A P=B P+P C$, if and only if the sides have the form $a=m u^{2}+n v^{2}$, $b=u(m u+n v), c=v(m u+n v)$. Here $u$ and $v$ are positive real numbers and $m / n$ is the ratio in which $A P$ divides the side $B C$.

Proof. We refer to Fig. 4. From $(*)$ we have

$$
A D^{2}=\frac{m b^{2}+n c^{2}}{m+n}-\frac{m n a^{2}}{(m+n)^{2}} .
$$

Since the triangles $B D P$ and $A D C$ are similar, we find that

$$
\frac{B D}{A D}=\frac{B P}{A C}, \text { i.e., } \quad B P=\frac{m a b}{(m+n) A D} .
$$




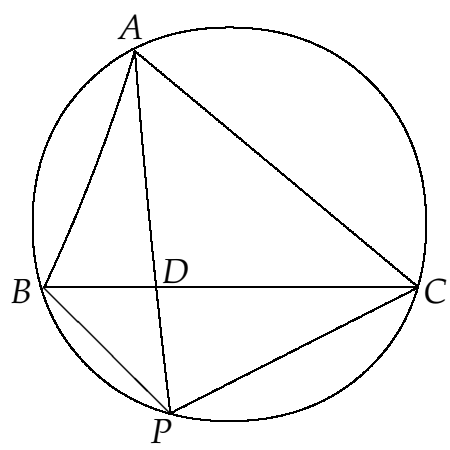

Fig. 4 When $A P=B P+P C$ ?

Likewise from the similar triangles $C D P$ and $A D B$ we find that

$$
P C=\frac{n a c}{(m+n) A D} .
$$

Hence $A P=B P+P C$, if and only if

$$
\begin{aligned}
A D \cdot A P & =\frac{m a b}{m+n}+\frac{n a c}{m+n}, \quad \text { or } \\
A D^{2}+A D \cdot D P & =\frac{a(m b+n c)}{(m+n)} .
\end{aligned}
$$

In Fig. $4, A P$ and $B C$ are intersecting chords of the circle. Therefore, $A D \cdot D P=$ $B D \cdot D C=m n a^{2} /(m+n)^{2}$. Using the preceding result and substituting (1) into (2) gives

$$
a(m b+n c)=m b^{2}+n c^{2} .
$$

Hence, the sides $a, b, c$ of $\triangle A B C$ must be expressible as $a=\left(m b^{2}+n c^{2}\right) /(m b+n c)$, $b, c$. We now take $b=u, c=v$ as arbitrary positive real numbers and multiply the sides by $(m u+n v)$. This similarity transformation enlarges $A P, B P, P C$ also by the same factor and hence the property $A P=B P+P C$ still holds. This gives the characterization mentioned in Theorem 1.

We now deduce a number of corollaries from Theorem 1. Corollary 1 gives another but simpler necessary and sufficient condition for $\triangle A B C$ to have the desired point $P$.

Corollary 1 Let $\triangle A B C$ be inscribed in a circle. A point $P$ exists on the minor $\overparen{B C}$ such that $A P=B P+P C$, if and only if the sides $a, b, c$ satisfy the inequalities $b>a>c$ or $c>a>b$.

Proof. Theorem 1 tells us that the desired point $P$ exists if and only if $a=\left(m b^{2}+\right.$ $\left.n c^{2}\right) /(m b+n c)$. This is equivalent to $m / n=c(c-a) / b(a-b)$ or $a=b=c$, the latter being excluded here. Since $A P$ intersects $B C$ internally, the ratio $m / n>0$. This is possible if and only if $a, b, c$ satisfy $b>a>c$ or $c>a>b$ and the proof is complete.

In fact, Corollary 1 provides greater insight into the subject under discussion as we see below. 
Corollary 2 A non equilateral isosceles triangle $A B C$ does not contain a point $P$ on the minor $\overparen{B C}$ such that $A P=B P+P C$.

Proof. The hypothesis of Corollary 2 implies exactly one of $a=b, a=c, b=$ $c$. This is neither $b>a>c$ nor $c>a>b$. Hence, $P$ does not exist. Alternatively, Theorem 1 shows that if one of the three above equalities holds then the other two must follow. This forces $\triangle A B C$ to be equilateral contradicting the hypothesis of Corollary 2.

Corollary 3 shows the uniqueness of the point $P$.

Corollary 3 If the sides of $\triangle A B C$ satisfy $b>a>c$ or $c>a>b$, then there exists $a$ unique point $P$ on the minor $\overparen{B C}$ such that $A P=B P+P C$.

Proof. In the proof of Corollary 1 we saw that $m / n=c(c-a) / b(a-b)$. Hence the ratio $\mathrm{m} / \mathrm{n}$ is uniquely determined by $a, b, c$. Therefore, the point $D$ on $B C$ and hence $P$ are unique, too.

Corollary 4 Suppose that a set of points $P_{i}$ exists on the minor $\overparen{B C}$ of $\triangle A B C$ such that $A P_{i}=B P_{i}+P_{i} C, i=1,2, \ldots$ Then, $\triangle A B C$ must be equilateral.

Proof. Since $\triangle A B C$ is fixed, $a, b, c$ remain constant but the ratio $m / n$ varies as $P$ moves over $P_{i}$. In other words, the equation $m b(a-b)=n c(c-a)$ can hold for many pairs of values of $m, n$, if and only if $a=b$ and $c=a$ implying that $\triangle A B C$ must be equilateral.

Corollary 5 (Hoppe triangles) Let $A P$ (Fig. 4) bisect $\angle B A C$ of $\triangle A B C$. Then, $A P=$ $B P+P C$, if and only if the sides $b, a, c$ are in arithmetic progression.

Proof. The angle bisector theorem states that $A P$, i.e., $A D$ bisects $\angle B A C$, if and only if $m / n=c / b$. Hence, $A P=B P+P C$, if and only if $a=\left(m b^{2}+n c^{2}\right) /(m b+n c)=\frac{1}{2}(b+c)$. This establishes the corollary. In fact, here $A P=2 B P$ and $A D=\frac{1}{2} \sqrt{3 b c}$.

Remark 2 Dickson in his monumental work, History of the Theory of Numbers [1] tells that Hoppe gives a complete discussion of Heron triangles (triangles with integer sides and area) in which the sides are in arithmetic progression. To honour Hoppe we call a triangle Hoppe triangle if the sides are in arithmetic progression. At this point we mention that there is a vast literature on Heron triangles and the reader is invited to refer to $[3,4,6,7]$ to get a glimpse of the many interesting Heron problems.

Corollary 6 Suppose the elements $a, b, c, m, n$ of $\triangle A B C$ are positive integers. If $A D$ is a median of $\triangle A B C$, then $A P=B P+P C$, if and only if $A B+A C$ is either a square or twice a square.

Proof. Since $A D$ is a median it follows that $B D=D C$ and $m=n=1$. Therefore, $a=u^{2}+v^{2}, b=u(u+v), c=v(u+v)$. Now, $A B+A C=(u+v)^{2}$ is a (perfect) square. However, if $u$ and $v$ are both odd integers, then $\operatorname{gcd}(a, b, c)=2$. The sides of the reduced (but similar) triangle will have $A B+A C$ twice a square. Conversely, if $A B+A C=w^{2}$ or $2 w^{2}$, then we can always choose $v=1, u=w-1$ to get a desired $\triangle A B C$. 
An example. To make the argument clearer, suppose $A B+A C=36=6^{2}$. Then, $u+v=6$. If $u=5, v=1$, then $a=26, b=30, c=6$ and $\operatorname{gcd}(a, b, c)=2$. If we divide the sides by 2 , then the reduced similar $\triangle A B C$ has sides $a=13, b=15$, $c=3$ and $A B+A C=18=2(3)^{2}$ is twice a square. We remark that under a similarity transformation, the property $A P=B P+P C$ is invariant.

Next, we consider the right angled triangle analogue mentioned in the introduction. Theorem 2 provides a characterization of $\triangle A B C$ in which there is a point $D$ on the side $B C$ such that $A D^{2}=B D \cdot D C$. In other words, $A D$ is the geometric mean between $B D$ and $D C$.

Theorem 2 In triangle $A B C$ a point $D$ on the side $B C$ exists with the property $A D^{2}=$ $B D \cdot D C$, if and only if the sides $a, b, c$ satisfy the inequalities $\sqrt{2} a \geq b+c>a$.

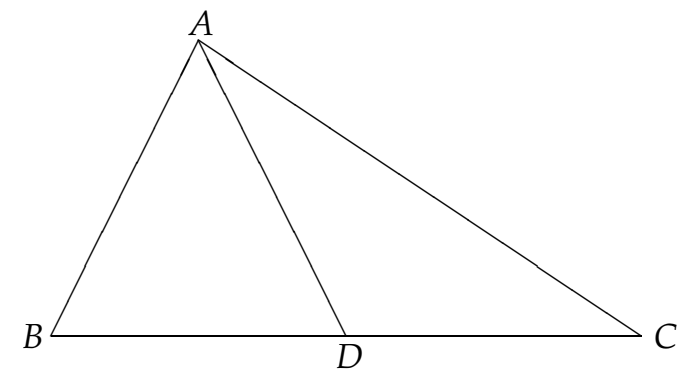

Fig. 5 When is $A D$ the geometric mean of $B D$ and $D C$ ?

Proof. We refer to Fig. 5. From $(*)$ the equation $A D^{2}=B D \cdot D C$ is equivalent to

$$
\frac{m b^{2}+n c^{2}}{(m+n)}-\frac{m n a^{2}}{(m+n)^{2}}=\frac{m n a^{2}}{(m+n)^{2}} .
$$

We rewrite the above equation as a quadratic in $m / n$. This yields

$$
\left(\frac{m}{n}\right)^{2} b^{2}+\left(b^{2}+c^{2}-2 a^{2}\right)\left(\frac{m}{n}\right)+c^{2}=0
$$

with the solutions

$$
\frac{m}{n}=\frac{-b^{2}-c^{2}+2 a^{2} \pm \sqrt{\left((b+c)^{2}-2 a^{2}\right)\left((b-c)^{2}-2 a^{2}\right)}}{2 b^{2}} .
$$

By the triangle inequality it follows that $b-c<a$. Hence, $(b-c)^{2}-2 a^{2} \leq 0$. To get a real root for $m / n$ we must therefore have $(b+c)^{2}-2 a^{2} \leq 0$. That is $\sqrt{2} a \geq b+c>a$, the characterization asserted by the theorem.

Remark 3 In general, equation (3) yields two roots for $m / n>0$ and hence two positions for $D$ on $B C$ such that the cevian $A D$ is the geometric mean of $B D$ and $D C$. $D$ exists uniquely when $m / n$ is a double root. This happens, if and only if $\sqrt{2} a=b+c$. Corollary 7 tells more about such triangles. 
Corollary 7 The point $D$ on side $B C$ of $\triangle A B C$ exists uniquely with the property $A D^{2}=B D \cdot D C$, if and only if $A D$ bisects $\angle B A C$.

Proof. In Remark 3 we saw that $D$ exists uniquely, if and only if $\sqrt{2} a=b+c$. In this case, equation (3) shows that $m / n=\left(-b^{2}-c^{2}+2 a^{2}\right) / 2 b^{2}=c / b$. The angle bisector theorem says that $m / n$, i.e., $B D / D C=c / b$, if and only if $A D$ bisects $\angle B A C$. This establishes Corollary 7. Moreover, in this case we find that $A D=\sqrt{\frac{1}{2} b c}$.

Corollary 8 gives the length of the tangent at the vertex $A$ to the circle $A B C$ intercepted by the side $B C$.

Corollary 8 Let $\triangle A B C$ be inscribed in a circle. Suppose the tangent at $A$ meets the side $B C$ (extended) at $D$. Then, $A D=a b c /\left|c^{2}-b^{2}\right|$.

Proof. The tangent-secant property says that $A D^{2}=B D \cdot D C$. If $c>b$, then $D$ lies to the right of $B C$ and if $c<b$, then $D$ lies to the left of $B C$. If $c=b$, then $A D$ is parallel to $B C$ and undefined as a length. In either case, $B D / D C=\left|c^{2} / b^{2}\right|$. Hence, $B D=c^{2} a /\left|c^{2}-b^{2}\right|, D C=b^{2} a /\left|c^{2}-b^{2}\right|$ and the assertion of the corollary follows.

\section{Conclusion}

The reader witnessed an interesting discussion from the consideration of analogies of known results. Euclidean geometry has been studied for thousands of years and yet new results appear on the pages of mathematics journals around the world. We invite the reader to make a new contribution to the following lovely but unsolved problem in natural number solutions (as far as I am aware) on the equilateral triangle:

Let $P$ be a point in the plane of an equilateral triangle $A B C$. Assume that $P A=p$, $P B=q, P C=r$ are natural numbers. Determine the length $s$ in natural numbers of a side of $\triangle A B C$ in terms of $p, q, r$.

This is a famous problem with rich history. Reference [2] gives the details. The numbers $p, q, r, s$ are inter-related by the equation

$$
3\left(p^{4}+q^{4}+r^{4}+s^{4}\right)=\left(p^{2}+q^{2}+r^{2}+s^{2}\right)^{2} .
$$

The remarkable symmetry exhibited by the above equation shows that any three of the four values $p, q, r, s$ may be taken as the distances of the point $P$ and the fourth one gives the side length of the equilateral triangle. The natural number solutions for $p, q, r$, $s$ are known in the special case when the point $P$ lies on a side or on the circum circle of the equilateral $\triangle A B C$. These two instances are covered by setting $p=q+r$ and then solving for $s$. Another special but new solution is found in [5]. Who is the lucky person who finds the general solution of the above problem/equation in natural numbers?

Acknowledgement. The author thanks Ms Christa Dobers who kindly produced the LaTEX version of my hand written paper. 


\section{References}

[1] Dickson, L.E.: History of the Theory of Numbers. Vol. II, Chelsea, New York 1971, 171-201.

[2] Mitrinović, D.S. et al.: Recent Advances in Geometric Inequalities. Kluwer Academic Publishers, Dordrecht 1989, 391-392 (Chapter XIII).

[3] Sastry, K.R.S.: Heron angles. Mathematics and Comp. Education 28 (1994), 24-35.

[4] Sastry, K.R.S.: Heron triangles: a new perspective. Aust. Math. Soc. Gazette 26 (1999), 160-168.

[5] Sastry, K.R.S.: Natural Number Solutions to $3\left(p^{4}+q^{4}+r^{4}+s^{4}\right)=\left(p^{2}+q^{2}+r^{2}+s^{2}\right)^{2}$. Mathematics and Comp. Education 34 (2000), 6-11.

[6] Sastry, K.R.S.: Heron Triangles: A Gergonne-Cevian-and-Median Perspective. Forum Geometricorum 1 (2001), 17-24.

[7] Sastry, K.R.S.: Heron Ladders. Math. Spectrum 34 (2001/2002), 61-64.

[8] Sastry, K.R.S.: Brahmagupta quadrilaterals: a description. Crux Mathematicorum with MM (to appear).

K.R.S. Sastry

Jeevan Sandhya

Doddakalsandra Post

Raghuvana Halli

Bangalore 560 062, India 